\title{
Integrity of the core mitochondrial RNA-binding complex 1 is vital for trypanosome RNA editing
}

\author{
ZHENQIU HUANG, ${ }^{1,2}$ DRAHOMÍRA FAKTOROVÁ, ${ }^{1,2}$ ADÉLA KŘíŽOVÁ, ${ }^{2}$ LUCIE KAFKOVÁ, ${ }^{3}$ LAURIE K. READ, ${ }^{3}$ \\ JULIUS LUKEŠ, ${ }^{1,2,4}$ and HASSAN HASHIMI ${ }^{1,2}$ \\ ${ }^{1}$ Biology Center, Institute of Parasitology, Czech Academy of Sciences, České Budějovice (Budweis), 370 05, Czech Republic \\ ${ }^{2}$ Faculty of Sciences, University of South Bohemia, České Budějovice (Budweis), 370 05, Czech Republic \\ ${ }^{3}$ Department of Microbiology and Immunology, School of Medicine, State University of New York at Buffalo, Buffalo, New York 14214, USA \\ ${ }^{4}$ Canadian Institute for Advanced Research, Toronto, Ontario M5G 1Z8, Canada
}

\begin{abstract}
Trypanosoma brucei is the causative agent of the human and veterinarian diseases African sleeping sickness and nagana. A majority of its mitochondrial-encoded transcripts undergo RNA editing, an essential process of post-transcriptional uridine insertion and deletion to produce translatable mRNA. Besides the well-characterized RNA editing core complex, the mitochondrial RNA-binding 1 (MRB1) complex is one of the key players. It comprises a core complex of about six proteins, guide RNA-associated proteins (GAPs) 1/2, which form a heterotetramer that binds and stabilizes gRNAs, plus MRB5390, MRB3010, and MRB11870, which play roles in initial stages of RNA editing, presumably guided by the first gRNA:mRNA duplex in the case of the latter two proteins. To better understand all functions of the MRB1 complex, we performed a functional analysis of the MRB8620 core subunit, the only one not characterized so far. Here we show that MRB8620 plays a role in RNA editing in both procyclic and bloodstream stages of $T$. brucei, which reside in the tsetse fly vector and mammalian circulatory system, respectively. While RNAi silencing of MRB8620 does not affect procyclic $T$. brucei fitness when grown in glucose-containing media, it is somewhat compromised in cells grown in the absence of this carbon source. MRB8620 is crucial for integrity of the MRB1 core, such as its association with GAP1/2, which presumably acts to deliver gRNAs to this complex. In contrast, GAP1/2 is not required for the fabrication of the MRB1 core. Disruption of the MRB1 core assembly is followed by the accumulation of mRNAs associated with GAP1/2.
\end{abstract}

Keywords: RNA editing; mitochondrion; trypanosome

\section{INTRODUCTION}

In the kinetoplastid Trypanosoma brucei, 12 of 18 mitochondrial-encoded mRNAs undergo RNA editing for their maturation. In this process, uridines (U) are inserted into, or less frequently deleted from, specific positions in the transcript to decrypt open reading frames (ORFs), which subsequently serve as templates for the mitochondrial $(\mathrm{mt})$ translation machinery. Because most of resulting proteins are subunits of the mt respiratory chain, RNA editing is essential for the survival of $T$. brucei throughout its life cycle, in which it circulates between the insect vector and mammalian host (Schnaufer et al. 2001).

Small noncoding transcripts called guide (g) RNAs, ranging from 50 to 70 nucleotides (nts) in size, represent the informational component of RNA editing (Blum et al. 1990). A 5 -proximal region on the gRNA called the anchor domain

Corresponding author: hassan@paru.cas.cz

Article published online ahead of print. Article and publication date are at http://www.rnajournal.org/cgi/doi/10.1261/rna.052340.115. hybridizes to a cognate mRNA to be edited. The downstream information domain defines several editing sites (ESs) on the mRNA that undergo either a U-insertion or U-deletion event. When all the ESs have been edited, the information domain and mRNA are complementary via Watson-Crick and noncanonical $\mathrm{U}: \mathrm{G}$ base-pairing. A post-transcriptionally added $3^{\prime}$-oligo(U) tail on the gRNA likely stabilizes its interaction with mRNA during duplex formation (McManus et al. 2000).

Moreover, several protein complexes also play various essential roles in editing. The RNA editing core complex (RECC), also referred to as the 20S editosome, provides the requisite catalytic activities needed for $\mathrm{U}$-insertion/deletion at a given ES. One of three RECC endonucleases cuts the mRNA strand of the duplex at a base pair mismatch to yield

(C) 2015 Huang et al. This article is distributed exclusively by the RNA Society for the first 12 months after the full-issue publication date (see $\mathrm{http}: / /$ rnajournal.cshlp.org/site/misc/terms.xhtml). After 12 months, it is available under a Creative Commons License (Attribution-NonCommercial 4.0 International), as described at http://creativecommons.org/licenses/by$\mathrm{nc} / 4.0 /$. 
$5^{\prime}$ and $3^{\prime}$ fragments bridged by a gRNA (Carnes et al. 2008). An ES cut by the deletion site-specific endonuclease is processed by a $3^{\prime}$ to $5^{\prime}$ exonuclease, whose activity is restricted to the extra U's from the $5^{\prime}$ fragment (Ernst et al. 2009). If the ES is an insertion site, the RECC terminal U transferase (KRET2) appends the $5^{\prime}$ fragment with the titular nucleotide (Ernst et al. 2003). The mRNA encoding cytochrome $c$ oxidase (cox) 2 is cut by the third RECC endonuclease that recognizes this unique substrate, which contains a gRNA-like element in its $3^{\prime}$ UTR that guides the addition of 4 U's within the ORF by KRET2 (Golden and Hajduk 2005). After the appropriate editing event is finished at the ES, an RNA ligase reseals the two mRNA fragments (Schnaufer et al. 2001; Verner et al. 2015).

The cascade of core enzymatic steps encapsulated by RECC can be recapitulated in vitro for the editing of a single ES. However, the lack of RECC processivity in vitro suggests that essential components for editing progression are lacking. This aspect of RNA editing is especially important for pan-editing, the decryption of an ORF throughout a transcript with a $3^{\prime}$ to $5^{\prime}$ polarity as facilitated by multiple gRNAs (Maslov and Simpson 1992). We have proposed that these and other facets of in vivo RNA editing may be facilitated by another protein complex discovered after RECC that has been named the mitochondrial RNA-binding complex 1 (MRB1) (Hashimi et al. 2013). Its elucidated architecture shows that it is composed of a core complex and the TbRGG2 subcomplex (Ammerman et al. 2012). The MRB1 core is made up of six proteins with a still undefined stoichiometry. The gRNA-associated proteins (GAPs) 1 and 2 (also known as GRBC2 and 1, respectively) form a heterotetramer that binds and stabilizes these small transcripts (Weng et al. 2008; Hashimi et al. 2009). The GAP1/2 proteins also represent the only bona fide RNA-binding components of the complex identified to date. RNAi silencing of three other subunits called MRB5390, MRB3010, and MRB11870 (nomenclature by Ammerman et al. 2012) also impacts editing without destabilizing gRNAs, with editing stalling at initial stages of pan-editing, presumably guided by the first gRNA:mRNA duplex, in the case of the latter two proteins (Acestor et al. 2009; Ammerman et al. 2011, 2013).

Although there are complex protein-protein interactions between the TbRGG2 subcomplex and the MRB1 core, this association is greatly enhanced by the presence of RNA (Ammerman et al. 2012; Aphasizheva et al. 2014). This phenomenon may in part be due to the subcomplex containing TbRGG2 and one of the two highly similar paralogs MRB8170 and MRB4160, all of which bind RNA (Fisk et al. 2008; Kafková et al. 2012). The TbRGG2 subcomplex also contains a protein called MRB8180 (Ammerman et al. 2012), whose RNA-binding properties and functional analysis have still not been addressed. RNAi silencing of the subcomplex primarily impacts pan-edited mRNAs (Fisk et al. 2008; Ammerman et al. 2010; Kafková et al. 2012), lead- ing to the view that they are specific for transcripts requiring multiple gRNAs for their processing (Hashimi et al. 2013). The finding that TbRGG2 has RNA annealing and unwinding activities is consistent with this notion. Indeed, they could facilitate the formation of gRNA:mRNA duplexes, as well as melting them after the editing of all ESs encoded by given gRNA to allow the annealing of the next upstream gRNA on the cognate mRNA and/or facilitate the utilization of a single gRNA as editing proceeds through one gRNA-directed block (Ammerman et al. 2010; Foda et al. 2012).

In a highly concerted fashion, the MRB1 core, the TbRGG2 subcomplex, and RECC maturate mRNAs for their eventual translation into subunits of the $\mathrm{mt}$ respiratory chain. In the T. brucei procyclic stage (PS), living within the Glossina fly midgut, the cytochrome $c$-containing electron transport chain complexes III and IV pump protons out of the mt matrix to create electrochemical gradient across the inner membrane, which is used by the $\mathrm{F}_{\mathrm{O}} \mathrm{F}_{1}$-ATP synthase complex to generate ATP (Schnaufer et al. 2005). The reducing equivalents for this process are primarily supplied by the catabolism of proline in the mt matrix, although PS cells grown in glucose-containing SDM79 medium (Brun and Schönenberger 1979) circumvent this pathway, as they preferentially generate ATP via glycolysis (Coustou et al. 2008). This situation is reminiscent of the energy metabolism of the pathogenic slender T. brucei bloodstream stage (BS), which generates ATP exclusively by glycolysis.

Although BS T. brucei does not utilize the mt respiratory chain for energy generation and the cytochrome $c$-containing complexes III and IV are absent, the electrochemical gradient across the inner membrane is still required for cell viability. To achieve this condition, the ATP synthase consumes ATP to pump protons out of the mt matrix (Schnaufer et al. 2005; Brown et al. 2006). As such, pan-editing of the mRNA encoding ATP synthase subunit 6 (A6) is required for the complete assembly of this complex in BS (Hashimi et al. 2010). Interestingly, dyskinetoplastic (DK) strains of T. brucei that have lost the capacity for RNA editing exist in nature (Schnaufer et al. 2005; Lai et al. 2008). The DK strains emerged independently several times (Carnes et al. 2005). To compensate for the loss of A6, these DK trypanosomes have acquired mutations in the ATP synthase $\mathrm{F}_{1}$-moiety $\gamma$-subunit, allowing their survival in the bloodstream of vertebrate hosts (Dean et al. 2013).

In this report, we describe the functional analysis of MRB8620 (TriTrypDB accession number: Tb927.11.16860; formerly "Tb11.01.8620"), the last remaining MRB1 core subunit as defined by Ammerman et al. (2012), by virtue of its consistent presence in various MRB1 core isolations, to be studied this way. This study was performed in the various physiological states of T. brucei in vitro cultures: PS grown in the presence and paucity of glucose and BS that has been rendered kDNA-independent due to bearing a $\gamma$-subunit site mutation that allows this condition. 


\section{RESULTS}

\section{MRB8620 interacts with other MRB1 subunits}

MRB8620 has been shown to interact with the MRB1 core complex and the TbRGG2 subcomplex by yeast two-hybrid analysis and various tandem affinity purification (TAPs) of tagged MRB1 subunits (Hashimi et al. 2008; Weng et al. 2008; Hernandez et al. 2010; Ammerman et al. 2011, 2012; Kafková et al. 2012). To confirm that MRB8620 is an integral component of the MRB1 complex, we analyzed proteins copurifying with the C-terminally PTP-tagged MRB8620 without nuclease treatment by LC-MS/MS mass spectroscopy. As shown in Table 1, MRB8620 interacts with all the other MRB1 core proteins (GAP 1/2, MRB5390, MRB3010, and MRB11870) plus subunits of the TbRGG2 subcomplex, and thus comprises an integral part of MRB1. Some common contaminants were also detected in the PTP eluates and are listed in Supplemental Table 1.

\section{MRB8620 associates with the inner mitochondrial membrane}

Using an updated gene model based on spliced leader trapping studies (Nilsson et al. 2010; Siegel et al. 2010), in silico-translated MRB8620 contains a predicted hydrophobic stretch of amino acids toward the $\mathrm{N}$-terminus that may serve as a transmembrane domain (Fig. 1A), as determined using the TMHMM 2.0 program (Möller et al. 2001). To test whether MRB8620 is indeed a membrane-associated protein, digitonin fractionated mitochondria isolated from the MRB8620-PTP-tag cell line were further processed into soluble and insoluble fractions by $0.1 \%$ Triton X-100. The resulting fractions were probed with antibodies against PTP-tag constituent protein A, HSP70 as an mt matrix marker, and respiratory complex IV subunit (trCOIV) as an inner membrane marker. As expected, HSP70 and trCOIV localized to the soluble matrix and insoluble membrane fractions, respectively (Fig. 1B). The protein A signal from the C-terminally tagged MRB8620, expressed from its endogenous locus by RNA polymerase II, is in the membrane fraction. Thus, MRB8620 is associated with the mt membrane.

\section{RNAi silencing of MRB8620 inhibits growth in glucose-poor media}

To facilitate the functional analysis of MRB8620 by reverse genetics, a PS cell line was generated that allows for the tetracycline-inducible expression of double-stranded (ds) RNA, which subsequently directs the RNAi machinery to degrade MRB8620 mRNA. To follow depletion of the protein, this cell line bears the MRB8620-PTP-tagged allele, which is also targeted by the dsRNA complementary to sequence within ORF. Upon depletion of MRB8620, no growth retardation was observed in the glucose-containing SDM-79 medium (Fig. 2A). The efficiency of RNAi was verified by Western blot analysis detecting the PTP-tagged protein via the a-protein A antibody (Fig. 2B). Indeed, MRB8620-PTP is considerably down-regulated 2 and $4 \mathrm{~d}$ after RNAi induction, although there is a slight recovery of its steady-state levels $6 \mathrm{~d}$ after tetracycline addition to the medium. Downregulation at the RNA level was also confirmed on Day 4 post-induction by qRT-PCR (Fig. 2C).

RNA editing is required for the maturation of mRNAs encoding several subunits of respiratory chain complexes, and the ablation of proteins involved in this process therefore negatively impacts oxidative phosphorylation in PS (Neboháčová et al. 2004; Zíková et al. 2006; Hashimi et al. 2010). Yet, oxidative phosphorylation does not contribute to energy metabolism when the T. brucei resides in SDM79,

TABLE 1. MRB8620-PTP associated proteins identified by mass spectrometry and ordered according to the number of unique peptides from PTP-tag elutions from two experiments

\begin{tabular}{|c|c|c|c|c|c|}
\hline Locus tag & Name $^{a}$ & Alias $^{b}$ & Subcomplex ${ }^{\mathrm{c}}$ & Unique peptides & Coverage \\
\hline Tb11.01.8620 & MRB8620 & TbGRBC3 & MRB1 core & 25 & $45 \%$ \\
\hline Tb11.02.5390 & MRB5390 & TbGRBC4 & MRB1 core & 54 & $38.6 \%$ \\
\hline Tb927.5.3010 & MRB3010 & TbGRBC6 & MRB1 core & 24 & $36.6 \%$ \\
\hline Tb927.4.4150/Tb927.8.8180d & MRB4150/MRB8180 & TbREMC4 & TbRGG2 & 21 & $25.8 \%$ \\
\hline Tb927.4.4160/ & MRB4160/ & TbREMC5/ & TbRGG2 & 20 & $24.6 \%$ \\
\hline Tb927.8.8170 & MRB8170 & TbREMC5A & & & \\
\hline Tb927.2.3800 & GAP1 & TbGRBC2 & MRB1 core & 18 & $33.7 \%$ \\
\hline Tb927.10.11870 & MRB11870 & TbGRBC5 & MRB1 core & 14 & $45.8 \%$ \\
\hline Tb927.7.2570 & GAP2 & TbGRBC1 & MRB1 core & 14 & $39.3 \%$ \\
\hline Tb927.7.800 & MRB800 & TbREMC3 & TbRGG2 & 10 & $19.3 \%$ \\
\hline Tb927.10.10130 & MRB10130 & TbREMC1 & None & 8 & $19.8 \%$ \\
\hline Tb927.10.10830 & TbRGG2 & & TbRGG2 & 5 & $24.1 \%$ \\
\hline
\end{tabular}

aNomenclature according to Ammerman et al. (2012).

${ }^{b}$ Nomenclature according to Aphasizheva et al. (2014).

${ }^{c}$ MRB1 subcomplex to which protein is assigned according to Ammerman et al. (2012).

${ }^{\mathrm{d}}$ Peptides that could have arisen from either of the two highly homologous proteins. 


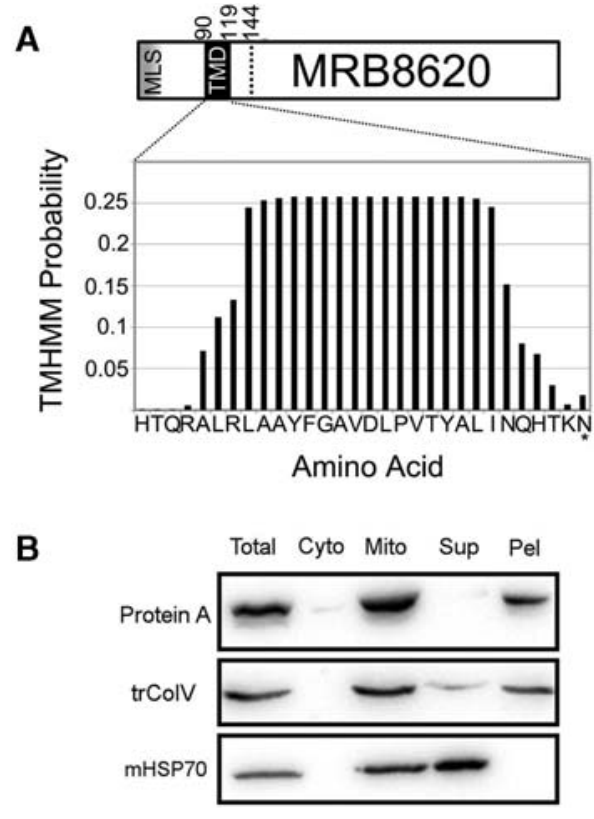

FIGURE 1. MRB8620 is a mitochondrial membrane-associated protein. (A) An N-terminal transmembrane domain (TMD) predicted in MRB8620. TMD predicted by TMHMM 2.0 program is located between the 90th and 119th amino acid sites with the probability of each residue being part of the TMD peaking $\sim 0.25$. Predicted N-terminal $\mathrm{mt}$ localization signal (MLS) shown on the left. A stretch of amino acids with small probability of being part of a TMD extends to the 144th amino acid (dotted line) with the same score as the amino acid marked with the asterisk. Numbering of amino acid residues is from the starting methionine. (B) Western blot analysis of digitonin fractionation of cytoplasm (Cyto) and mitochondrial (Mito), as well as Triton X-100 fractionation of $\mathrm{mt}$ proteins into soluble and membrane fractions, containing in the supernatant (Sup) phases or pellet (Pel), respectively. Proportional volumes of each fraction were loaded as described in Material and Methods along with an equivalent amount of lysate from whole cells (Total) taken for comparison. Antibodies used are indicated on the left.

a medium containing ample glucose (Coustou et al. 2008). To determine whether any negative impact of MRB8620 depletion on parasite's fitness is masked in the energy-rich milieu of SDM79, the RNAi cell line was transferred to SDM80 medium, which does not contain glucose. The RNAi silencing of MRB8620 in SDM80 was verified on the protein (Fig. 2E) and RNA levels (Fig. 2F). A reproducible reduction in growth was observed after Day 4 of RNAi induction in a glucose-poor medium (Fig. 2D), with a doubling time from Days $4-6$ post-induction of $32.95 \pm 0.93 \mathrm{~h}$ in the MRB8620-silenced cells, versus $20.52 \pm 0.59 \mathrm{~h}$ in the noninduced controls. After $6 \mathrm{~d}$ post-induction, there is a recovery of the growth rate in MRB8620-depleted trypanosomes (22.55 $\pm 0.49 \mathrm{~h}$ doubling time at Days 6-8 versus $20.87 \pm$ $0.26 \mathrm{~h}$ at Days $8-10)$, although it still lags when compared with the noninduced controls $(19.66 \pm 1.02 \mathrm{~h}$ doubling time at Days 6-8 versus $18.87 \pm 0.86 \mathrm{~h}$ at Days $8-10)$. Thus, down-regulation of MRB8620 inhibits growth under glucose-poor conditions, in which oxidative phosphorylation is required for energy production.

\section{Ablation of MRB8620 affects editing in procyclic stage}

Upon RNAi silencing of MRB8620, the growth of PS is affected in SDM80 as opposed to SDM79. Hence, we investigated the effect of MRB8620 silencing on steady-state levels of mt RNAs under both growth conditions, in order to explain lower fitness of PS flagellates under conditions in which they depend on a functional respiratory chain for energy generation.

To address this question, RNA was harvested from uninduced and MRB8620 RNAi-induced cells grown in glucose-poor SDM80 for $4 \mathrm{~d}$. Some of the RNA was converted to cDNA by reverse transcription to serve as a template for the qPCR assay of mt mRNA and rRNA steady-state levels (Carnes et al. 2005). Figure 3A depicts the relative abundances of the assayed transcripts in the RNAi silenced cells when compared with the noninduced controls. The neveredited 9S and 12S rRNAs as well as cytochrome $c$ oxidase 1 (CO1) and NADH dehydrogenase subunit 4 (ND4) mRNAs were not affected by MRB8620 ablation. From the mRNAs that require only modest $U$ insertions, called minimally edited, only cytochrome $c$ reductase subunit b (CYB) shows a $\sim 30 \%$ decrease and $\sim 20 \%$ increase in the edited and pre-edited species, respectively. The persistence of $\mathrm{CO} 2$ editing upon ablation of MRB8620 is notable, because it uniquely does not require a trans-acting gRNA for its editing, but uses a gRNA-like element within its $3^{\prime}$-UTR (Golden and Hajduk 2005). The pan-edited mRNAs, which require U insertion/deletion throughout the transcript, are most affected in the MRB8620 knockdowns (Fig. 3A). Furthermore, with the exception of ND7, there is a concomitant accumulation of pre-edited forms of the followed transcripts. Equivalent results were obtained from another clone examined in parallel (data not shown).

To eliminate the possibility that editing is affected because of the destabilization of gRNAs in the MRB8620-depleted trypanosomes, as was the case when GAP1 was RNAi-silenced (Weng et al. 2008; Hashimi et al. 2009), we assayed the steady-state levels of gRNAs by Northern blot analysis (Fig. 3B). In the tetracycline-treated cells, there was an almost twofold increase in the levels of gRNAs for the editing of ATP synthase subunit 6 (A6), mitochondrial unidentified reading frame 2 (MURF2), and $\mathrm{CO} 3$ when compared with the constant levels of the cytosolic 5.8S rRNA between the RNAiinduced and noninduced cells. Similar accumulation of these small transcripts has been previously observed when editing is impaired by knockdown of other MRB1 subunits (Ammerman et al. 2013; Aphasizheva et al. 2014).

After establishing that there is indeed a defect in RNA editing upon MRB8620 silencing in PS grown in glucose-poor SDM80, we decided to address whether such a phenotype is observed when the cells are grown in glucose-supplemented SDM79, a condition in which depletion of this MRB1 subunit does not affect cell growth. RNA harvested from these cells as before was converted to cDNA for the qPCR assay (Fig. 3C). 
A
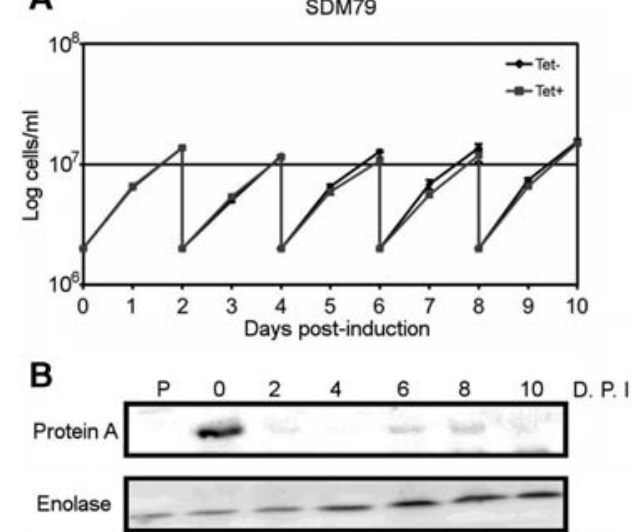

D
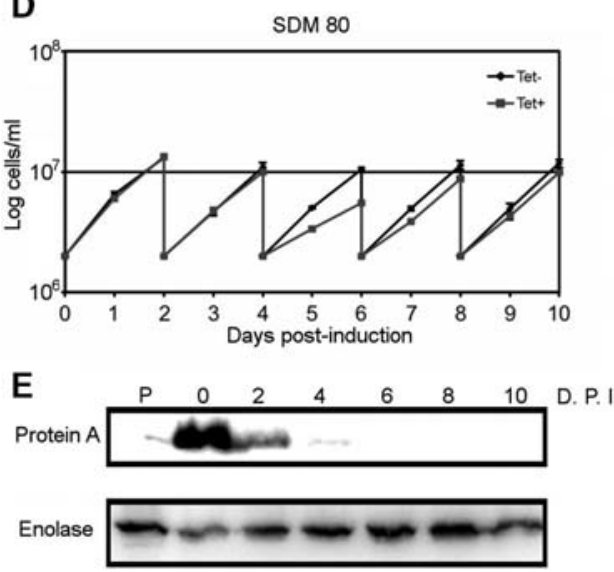

FIGURE 2. T. brucei growth is inhibited upon silencing of MRB8620 in glucose-poor SDM80 but not in glucose-containing SDM79. (A) Growth of PS cells in SDM79 in the presence (gray square and line) and absence (black diamond and line) of tetracycline (tet), which induces MRB8620 RNAi silencing, over a $10 \mathrm{~d}$ time course. The cells were diluted to the starting density of $2 \times 10^{6}$ cells/mL every $2 \mathrm{~d}$. (B) Western blot analysis of the RNAi silencing of MRB8620-PTP by a-protein A antibody, with $a$-enolase shown as a loading control. Cell lysates were collected from the parental cell line (P) as well as the MRB8620 knockdowns every even day 0 to $10 \mathrm{~d}$ post-induction (DPI) with tetracycline. $(C)$ Bar graph indicating the relative MRB8620 RNA levels in the RNAi-induced versus uninduced-control cells as determined by quantitative RT-PCR and normalized to $18 \mathrm{~S}$. Whiskers denote range of obtained relative abundancies within technical triplicates. $(D-F)$ The same as $A-C$ above, respectively, except that the cells were grown in SDM80.

Overall, these RNAs are affected similarly to those from the MRB8620 knockdown grown in SDM80: never-edited RNAs are not impacted, whereas minimally edited CYB and the assayed pan-edited mRNAs show the down-regulation of edited products and the concurrent accumulation of pre-edited transcripts.

To summarize this part, RNAi silencing of MRB8620 leads to a defect in RNA editing. There is a decrease in pan-edited transcripts as well as in the corresponding form of minimally edited CYB. This phenotype is accompanied by an accumulation of pre-edited transcripts, which are not processed when RNA editing is impeded, as well as gRNAs, suggesting that they may be turned over when utilized to guide editing events on an mRNA. This impact on RNA editing is independent of the energy metabolism state of the PS, although cell fitness is compromised only when the trypanosomes rely on oxidative phosphorylation for ATP generation. Subsequent experiments were performed on cells grown in SDM80.

\section{MRB8620 is required for MRB1 integrity}

As MRB8620 is a subunit of the MRB1 complex, we wondered whether its depletion by RNAi would affect the integrity of the whole complex. To this end, macromolecules isolated from tetracycline-induced MRB8620 and GAP1 RNAi cells in addition to the parental line from which they were derived were separated on a $10 \%-30 \%$ glycerol gradient by ultracentrifugation. Twelve fractions of increasing density were probed with antibodies recognizing MRB1 core subunits GAP1 and MRB3010, as well as TbRGG2 (Ammerman et al. 2012). The RECC subunit KREPA3 was also immunodecorated to serve as a marker for 20 Svedberg (S) sedimentation within the gradient, taking advantage of this biochemical property of the complex (Golas et al. 2009). As shown in Figure 4A, the ablation of MRB8620 affects the distribution of GAP1 and MRB3010 in the gradients (cf. parental and MRB8620 RNAi). In the case of GAP1, the protein is equally distributed throughout the gradient (fractions 1-21) upon MRB8620 silencing, which is in contrast to the pattern observed in the parental control. Signal from MRB3010 appears in lighter fractions than what was observed in the parental cell line (fractions 13-21), indicating that a significant fraction of this core subunit is notassembled into larger complexes. A very slight shift to lighter fractions was also observed for TbRGG2 (Fig. 4A). KREPA 3 mostly retained its characteristic $20 \mathrm{~S}$ sedimentation, indicating that the ablation of MRB8620 does not affect the integrity of RECC.

The observation that the sedimentation rate of the assayed MRB1 proteins is affected upon MRB8620 ablation prompted us to investigate intra-MRB1 interactions in this background. Toward this goal, we generated a cell line in which one of the MRB3010 alleles was in situ C-terminally tagged with the V5 epitope to facilitate the immunoprecipitation (IP) of the respective protein by a cross-reacting $\alpha$-V 5 antibody. In this way, we were able to determine which proteins co-IP with MRB3010-V5 in the presence and absence of 

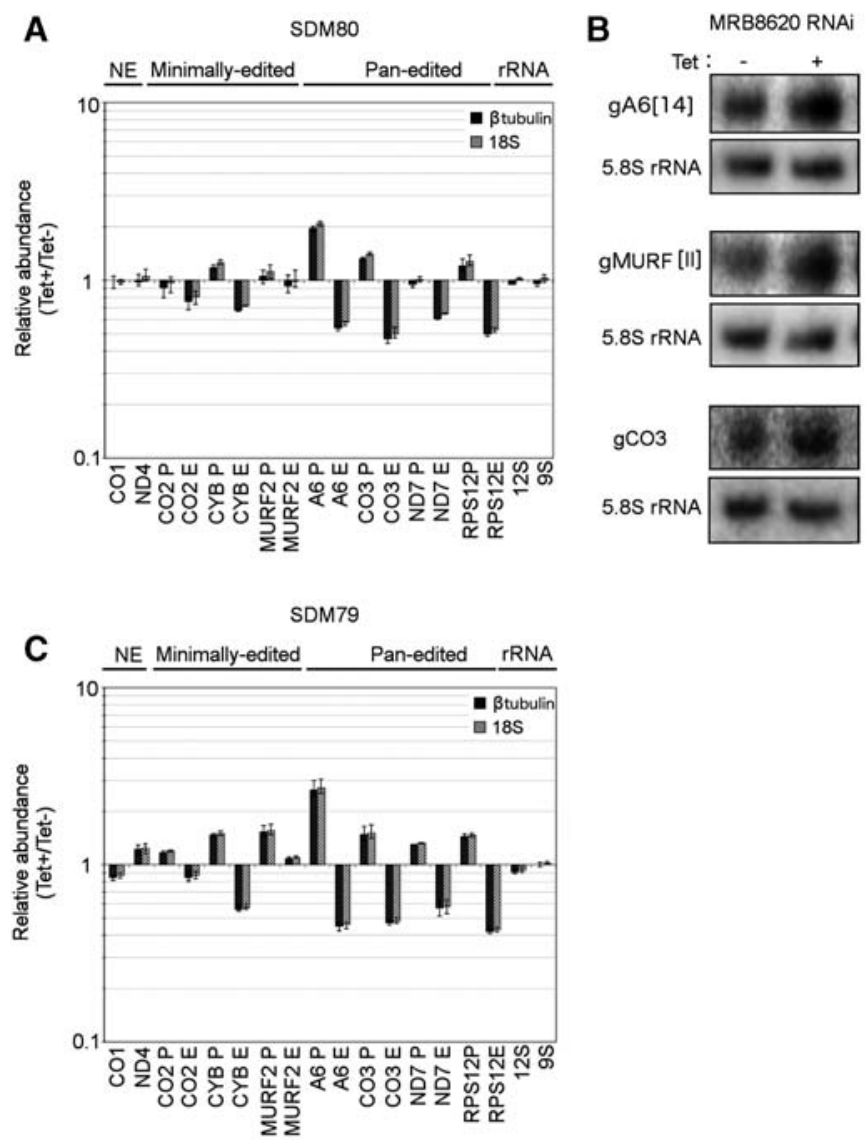

FIGURE 3. MRB8620 plays a role in RNA editing. (A) RNA was isolated from PS cultured in SDM80 to Day 4 post-induction. RNA abundance was quantified by qRT-PCR using primer pairs specific for selected never-edited, minimally edited, pan-edited, and ribosomal RNAs, as labeled on the $x$-axis. The bar graph depicts the relative abundance of RNA levels in tetracycline-induced T. brucei compared to uninduced-control cells. RNA levels were standardized to $\beta$-tubulin (black bar) or $18 \mathrm{~S}$ (striped bar) cDNA. Whiskers denote range of obtained relative abundances within technical triplicates. (B) Levels of gRNAs in MRB8620 RNAi T. brucei grown in SDM80. Nylon membranes were probed with $5^{\prime}$ end labeled oligonucleotides complementary to gA6[14], gMURF2[II], or gCO3 gRNAs, with 5.8S rRNA set as a loading control for each membrane. The signals were quantified and normalized to the levels of 5.8S RNA from the same sample. The bar graph on the right depicts fold change in RNAi-induced cells compared with uninduced-control T. brucei and whiskers the error between duplicates. $(C)$ As in $A$, where the cells were cultured in SDM79. Top of the graph indicates whether species of RNA assayed is never-edited (NE), minimally, pan-edited, or mitochondrial rRNA. The following abbreviations were used for mRNAs assayed by qPCR: ATPase subunit 6 (A6), cytochrome $c$ oxidase subunits 1 (CO1), 2 (CO2), and 3 (CO3), cytochrome reductase subunit b (CYB), maxicircle unknown reading frame 2 (MURF2), NADH dehydrogenase subunits 4 (ND4) and 7 (ND7), and ribosomal protein S12 (RPS12). (P) Pre-edited RNA; (E) edited RNA.

MRB8620 (Fig. 4B). Interaction of MRB3010-V5 with GAP1 and MRB11870, another core MRB1 protein, is reduced when MRB8620 is down-regulated (Fig. 4B), which is consistent with the previous observation that in this background a portion of MRB3010 does not assemble into larger complexes and GAP1's sedimentation is affected. This experiment also indicates that the association of TbRGG2 and MRB8170, another member of the TbRGG2 subcomplex, is also reduced, albeit to a small degree in the former case (Fig. 4B). A decreased association of the TbRGG2 subcomplex with MRB3010-V5 in the MRB8620 knockdown is also in agreement with the apparent shift of TbRGG2 to lighter glycerol gradient fractions. The more dramatic decrease of MRB8170 association with MRB1 core upon MRB8620 depletion, compared with that of TbRGG2, is consistent with the strong direct interaction detected between MRB8170 and MR8620 in yeast two-hybrid studies (Ammerman et al. 2012.) The steady-state levels of the proteins examined in Figure 4 were not affected by the MRB8620 knockdown as evidenced by their equivalent signals in the loading of the glycerol gradients (Fig. 4A) or the IP inputs (Fig. 4B).

Because the integrity of the MRB1 core is compromised upon knockdown of its MRB8620 and MRB11870 subunits (Ammerman et al. 2013), we checked whether RNAi silencing of GAP1 has a similar phenotype in this respect. Unlike other core MRB1 proteins, GAP1/2 proteins form a heterotetrameric complex that binds gRNAs, the depletion of which leads to destabilization of these small, noncoding transcripts (Weng et al. 2008; Hashimi et al. 2009). Furthermore, GAP1/2 exhibits a heterodisperse distribution on glycerol gradients when compared with the other core subunits (Fig. 4A), suggesting that their interactions are not restricted to the MRB1 core. GAP1 RNAi did not result in a shift of MRB3010 or TbRGG2 to lighter fractions, the latter observation being agreement with a previous study (Aphasizheva et al. 2014), despite its demonstrated protein depletion (Fig. 4A, cf. GAP1 RNAi and parental). Next, we introduced a C-terminal V5-tagged MRB3010 allele into a PS cell line containing GAP1 RNAi construct (Hashimi et al. 2009). The IP of MRB3010-V5 was performed as with the MRB8620-depleted samples (Fig. 4C), and immunoprecipitated proteins probed with the same panel of antibodies. As expected, due to RNAi silencing, GAP1 was not detected in the MRB3010V5 IP (Fig. 4C). Interestingly, MRB11870 interaction with MRB3010-V5 persisted, perhaps even up-regulated, in the GAP1-depleted cells, indicating that GAP1 is not required for assembly of the MRB1 core. The extent of TbRGG2 and MRB8170 co-IP was equivalent in the GAP1-silenced cells and noninduced controls (Fig. 4C).

Thus, MRB8620 appears to be a central player in the integrity of the MRB1 core as well as core-TbRGG2 subcomplex 
A

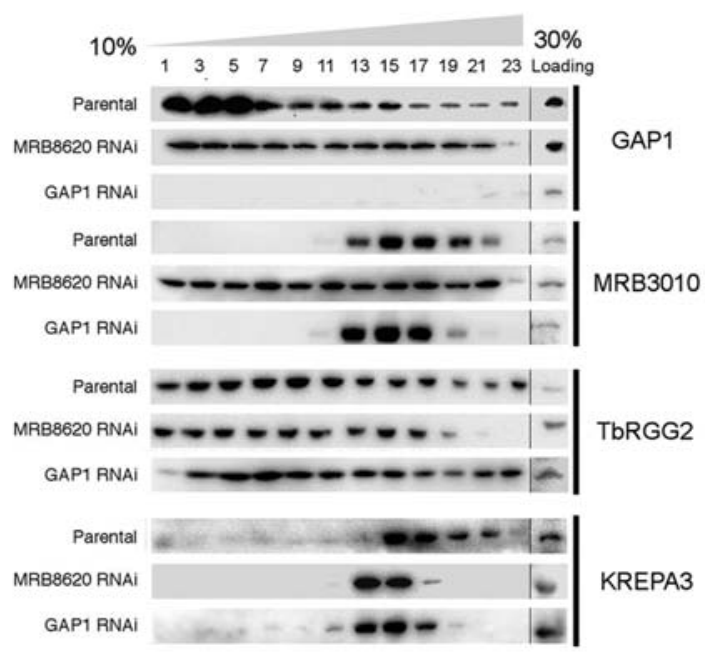

B
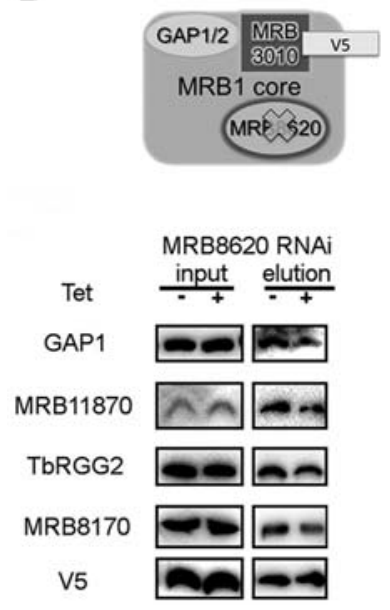

FIGURE 4. MRB8620 is required for MRB1 core integrity. ( $A$ ) The effect of MRB8620 and GAP1 depletion on the sedimentation of GAP1, MRB3010, TbRGG2, and RECC subunit KREPA3. Mitochondrial lysates from the parental cell line (top images), RNAi-silenced MRB8620 (middle images), and GAP1 (lower images) T. brucei were loaded on $10 \%-$ $30 \%$ glycerol gradients for their fractionation. Alternate gradient glycerol fractions were analyzed by immunodecoration of Western blots with the indicated antibodies indicated on the right. To the right of the 23rd fraction (loading) is a sample from the lysis step before loading onto the gradient to demonstrate equivalent amounts of protein were used among samples. (B) Scheme of MRB1 core complex components in cells harboring MRB8620 RNAi with MRB3010 V5 tagged at an endogenous allele. MRB3010-V5 and associated proteins were isolated by Protein GDynabead from extracts of mitochondria from either uninduced (tet-) or RNAi-induced (tet+) MRB8620 RNAi cells. Both input and elutions (indicated above images) were analyzed by Western blot for MRB1 complex components using the antibodies indicated on the right. (C) As in $B$, except that the cells harbor the GAP1 RNAi construct with MRB3010 V5 tagged at an endogenous allele.

interactions. Its ablation by RNAi results in a portion of the core subunit MRB3010 appearing in lower density fractions, indicating their compromised incorporation. This result was further confirmed by pull-down of MRB3010-V5 in a MRB8620-depleted background. In contrast, the MRB1 core can assemble in the absence of GAP1, because MRB3010-V5 interacts to the same degree with the other core protein MRB11870 upon RNAi silencing. Similar results were observed for interactions between core subunit MRB3010 and subunits of the TbRGG2 subcomplex in the presence and absence of MRB8620 and GAP1.

\section{Down-regulation of MRB8620 results in an accumulation of $m R N A s$ requiring editing associated with GAP1/2}

The integrity of MRB1 core is compromised upon RNAi silencing of MRB8620, eventually giving rise to disrupted RNA editing. We next investigated whether MRB8620 depletion affects the abundance of $\mathrm{mt}$ mRNAs associated with GAP1/2, given the loss of GAP1/2 association with the MRB1 core in this milieu. It has been shown that gRNAbound GAP1/2 heterotetramer is associated with mRNA bound protein (e.g., KREL1 and MERS1 [Weng et al. 2008]). This result indicates that edited mRNAs may associate with GAP1/2 subcomplex, given the annealed gRNA: mRNA duplexes as the substrate of RNA editing. The copurification of gRNAs and mRNAs with MRB3010 protein is precedent for this notion (Madina et al. 2014).

To assess the effect of MRB8620 depletion on GAP1/2mRNA association, we performed a GAP1 IP from mt lysates of MRB8620-silenced and untreated T. brucei (Fig. 5A). Dynabeads cross-linked with specific a-GAP1 antibody (Hashimi et al. 2009) were used. IP of GAP1 was equally efficient from both RNAi-induced and untreated samples, as shown by Western blot analysis using the a-GAP1 antibody (Fig. 5A). RNA isolated from the IP eluates was converted into cDNA to serve as a template for the aforementioned real-time qPCR assay for mt mRNA levels (Carnes et al. 2005).

To demonstrate the enrichment of mRNA from the GAP1 IP, a comparison was performed between mRNA precipitated from antibody-conjugated Dynabeads and that from blank beads as a mock. As performed in a previous study (Madina et al. 2014), we confirmed that cDNAs from $\mathrm{mt}$ RNAs were enriched in the GAP1 IP when compared with the mock (Supplemental Fig. 1). Interestingly, pre-edited and edited A6, CO3, and ND7 were more abundant in the GAP1 IP when compared with the mock, whereas the never-edited mt mRNAs CO1 and ND4, plus nuclear $18 \mathrm{~S}$ rRNA and cytosolic $\beta$-tubulin mRNA, were equally represented in both samples. Thus, GAP1 associates with mRNAs that hybridize with gRNAs for their maturation.

Next, we compared the amount of pre-edited and edited mRNAs pulled down by GAP1 IP in the presence and down-regulation of MRB8620 by relative qPCR quantification (Pfaffl 2001). Consistent with never-edited CO1 and ND4 RNAs being carried over during the IP procedure, cDNAs representing these transcripts were equally present in the RNAi-silenced and untreated samples (data not shown) and thus were used to normalize the relative abundancies of 


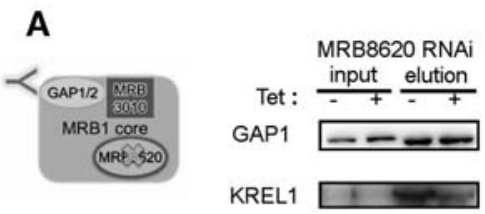

B

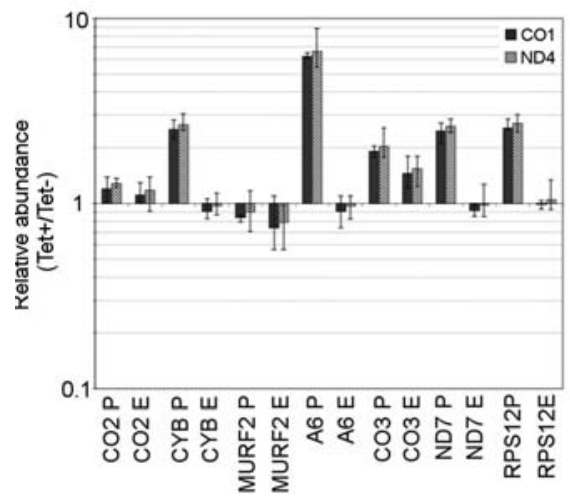

C
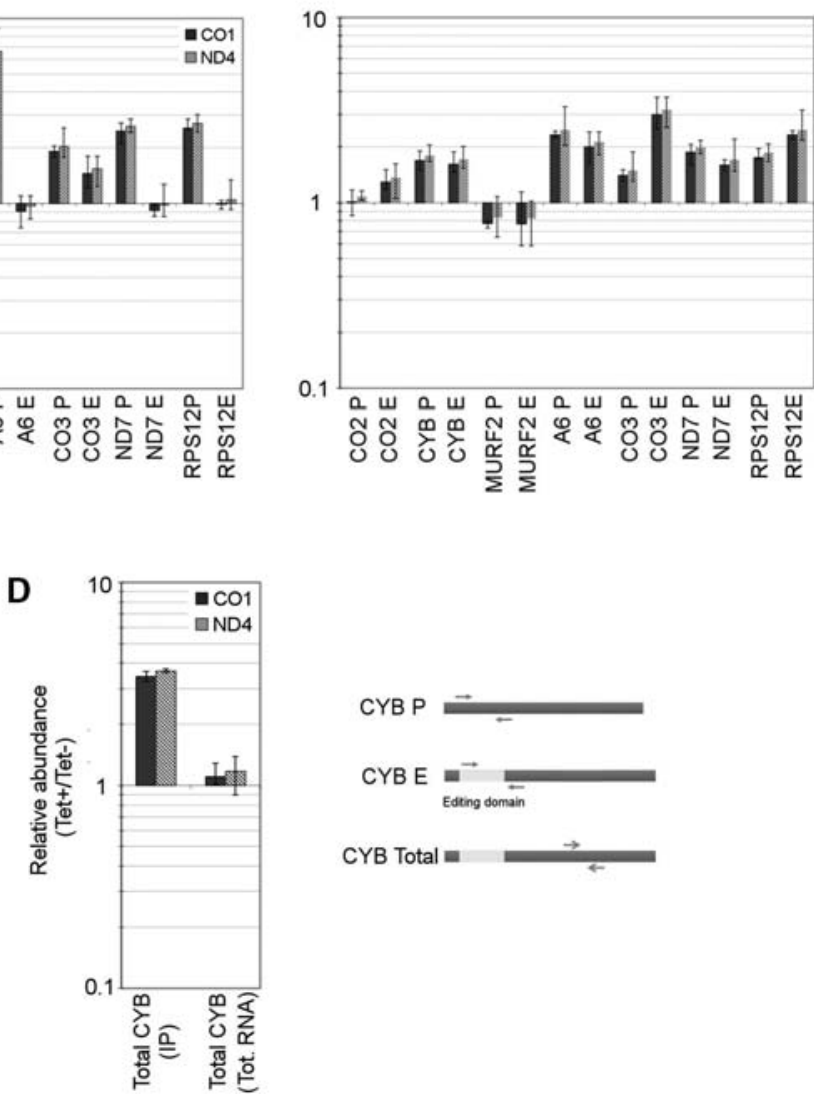

FIGURE 5. GAP1/2 heterotetramer exhibits accumulation of mRNAs undergoing RNA editing and reduced association with RECC upon MRB1 core disassembly by MRB8620 RNAi. $(A)$ Scheme depicting GAP1/2 IP in MRB8620 RNAi-silenced cells (left). Western blot (right) of GAP1 IPs performed in MRB8620 RNAi-induced (Tet+) and uninduced cells (Tet-) were immunodecorated with antibodies against GAP1 and RECC subunit KREL1. (B) GAP1/2-bound RNAs isolated from the elutions from $A$ were converted to cDNA for quantification by real-time PCR with selected primer sets for minimally edited and pan-edited RNAs. The bar graph indicates fold changes in RNA isolated from MRB8620 RNAi-induced cells compared to that from uninduced cells normalized to never-edited mRNAs CO1 (black bars) and ND4 (striped bars). Whiskers denote range of obtained relative abundances within technical triplicates. $(C)$ Relative abundance of pre-edited and edited mRNAs pulled down in GAP1/2 IPs as in $B$ but when normalized to the levels of the same transcripts from total RNA (Fig. 3A) in MRB8620-depleted and uninduced controls. This normalization is elaborated in Materials and Methods. $(D)$ Relative abundance of total CYB transcripts pulled down with GAP1 in the IP as well as total RNA (Tot. RNA) from MRB8620-depleted and uninduced controls, and depicted as in $B$. The scheme on the right in $D$ indicates the primers designed to detect the pre-edited (P), edited (E), and total CYB transcripts. All qPCR graphs are representative data from one of two IPs.

the assayed pre-edited and edited transcripts. Figure 5B reveals a consistent accumulation of pre-edited transcripts associated with GAP1 in the MRB8620 knockdown. Such a buildup of pre-edited mRNAs is consistent with the hypothesis that RNA editing is blocked upon MRB8620 depletion.
Edited mRNAs are associated with GAP1 in equal measure in the presence or ablation of MRB8620, with the exception of $\mathrm{CO} 3$, which shows an accumulation. Because the levels of many RNAs differ in the input samples in response to MRB8620 depletion, we next normalized the relative levels of RNAs associating with GAP1 in the knockdown and control cells to their relative levels as assayed from total RNA isolated from the same sample types (Fig. 3A). As seen in Figure 5C, the enrichment of pre-edited mRNAs persists when the data are processed in this way. In addition, we observed that the relative abundance of many edited mRNAs associating with GAP1 also appears to be enriched in MRB8620 knockdown versus control cells, suggesting that the small amount of edited RNAs present in these cells also accumulates in association with GAP1/2 when MRB8620 is depleted. The exceptions to this pattern are the preedited and edited forms of MURF2, which exhibit a decrease in association with GAP1 upon MRB8620 depletion.

To circumvent the complication of changing RNA levels in the input in MRB8620-depleted cells, we next measured total CYB mRNA, using $\mathrm{qPCR}$ primers annealing to the CYB mRNA sequence outside of its short editing domain (Fig. 5D), which are expected to reflect the combined relative abundance of pre-edited, edited transcripts and intermediates. These primers were utilized on the cDNA generated from the GAP1 IP eluates as well as the total RNA in the knockdown and untreated controls, as described thus far in this section. Figure $5 \mathrm{D}$ shows that CYB mRNA measured using this primer pair was $\sim 2.5$-fold enriched in GAP1 IPs in MRB8620 knockdown cells compared with control cells, despite the constant levels measured in total RNA. From these data and those in Figure 5B,C, we conclude that both preedited and edited mRNAs accumulate in association with GAP1/2 when the MRB1 core is disrupted by MRB8620 depletion.

The accumulation of pre-edited $\mathrm{mt}$ mRNAs in association with GAP1 is consistent with an impairment in RNA editing. As the GAP1 heterotetramer has been demonstrated to associate with RECC via RNA linkers (Aphasizheva et al. 2014), and the latter complex confers the catalytic activities needed 
for RNA editing, we assayed whether RECC and GAP1/2 associate upon MRB8620 silencing. Western blots of GAP IP eluates from MRB8620-down-regulated and untreated cells were immunodecorated with the KREL1 protein to serve as marker for RECC as it represents one of the RNA ligases of the complex (Schnaufer et al. 2001). This signal is weaker in the MRB8620 knockdown (Fig. 5A), suggesting that RECC association with GAP1/2 is compromised in this background, leading to an accumulation of preprocessed RNAs bound to GAP1/2.

In summary, the GAP1/2 heterotetramer associates with mRNAs that require RNA editing for their maturation. When the MRB1 core is disrupted upon MRB8620 silencing, there is an accumulation of mRNAs undergoing editing in association with GAP1/2. Furthermore, there is also a decreased association between the immunoprecipitated GAP1/ 2 and RECC, which provides the core enzymatic activities needed for RNA editing.

\section{MRB8620 is necessary for editing in bloodstream stage $T$. brucei}

Our observation that RNAi silencing of MRB8620 impacts RNA editing in PS, regardless of the metabolic status of the cells, contradicts a previous report (Aphasizheva et al. 2014). To verify this phenotype, we set out to determine whether MRB8620 is also required for RNA editing in BS. Our initial attempts to create a double MRB8620 gene knockout (DKO) in BS failed (data not shown), suggesting that this protein is essential for the parasite.

Given this complication, we opted to take advantage of a BS cell line bearing a L262P site mutation in the ATP synthase $\gamma$-subunit, which allows T. brucei at this life-cycle stage to be viable despite blocked kDNA inheritance or expression (Dean et al. 2013; Schnarwiler et al. 2014). We predicted that the knockout of both MRB8620 alleles would be achievable as RNA editing is rendered redundant in this genetic background. After creating the cell line bearing the L262P site mutation in one of the $\gamma$-subunit gene alleles, we confirmed that this genetic modification indeed rendered this cell line kDNA-independent (see Materials and Methods).

To determine whether BS MRB8620 knockout cells exhibit RNA editing, we replaced both MRB8620 ORFs with constructs bearing the phleomycin and hygromycin resistance cassettes in L262P-mutant bearing BS, as shown by PCR with primer pair I annealing to genomic sequence just outside of the integration sites (Fig. 6A,B). More significantly, sequence within the MRB8620 ORF was not PCR amplified by primer pair II from genomic DNA extracted from the DKO (Fig. 6A,C). This amplicon was detected in the genomic DNA from BS in which only a single MRB8620 ORF was deleted and in the parental cell lines.

Demonstrating that we indeed generated a MRB8620 DKO, we harvested RNA from these cells and the parental L262P-mutant bearing cells in which both MRB8620 alleles
A
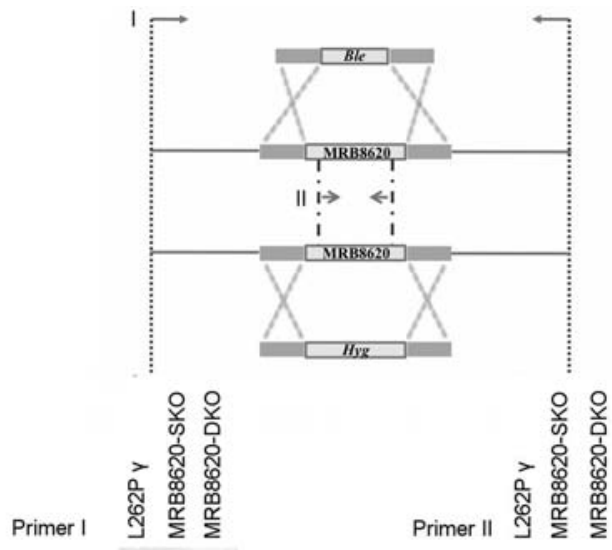

B
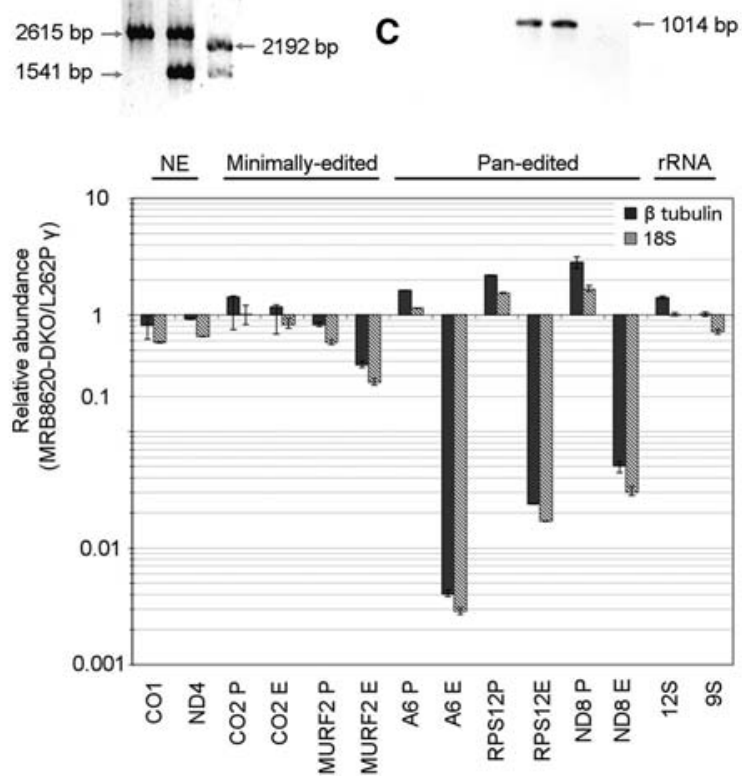

FIGURE 6. MRB8620 is essential for RNA editing in BS T. brucei. (A) Scheme depicting how MRB8620 double knockout was generated. Arrows indicate the locations of PCR primer pairs I and II, just flanking the knockout loci and amplifying the MRB8620 ORF, respectively, used to identify the single and double knockouts generated by homologous recombination in the cell line in which one of the ATP synthase $\gamma$-subunit alleles bears a L262P site mutation. (B) Agarose gel-resolved PCR products of primer pair I flanking the genomic integration site of the constructs from genomic DNA isolated from the parental hemizygous L262P-mutant BS T. brucei, single knockout (SKO) and MRB8620 double knockout (DKO). The size of each amplicon is indicated by the arrow. Primer pairs for identification are depicted in $A$. (C) As in $B$, but showing the PCR amplicon from primer pair II amplifying sequence from within the MRB8620 ORF. (D) Relative abundance of mitochondrial mRNAs in MRB8620 double knockout cells compared with the parental hemizygous ATP synthase L262P $\gamma$-subunit mutant cell line. Whiskers denote range of obtained relative abundances within technical triplicates. Labeled as in Figure 3A.

were intact, both grown under the same conditions. This material was converted to cDNA for use in the qPCR assay measuring steady-state levels of maxicircle-encoded RNAs. Neither never-edited CO1 and ND4 mRNAs nor 9S and $12 \mathrm{~S}$ rRNAs were affected in the DKO when compared with 
the parental cell line (Fig. 6D), indicating that maxicircle kDNA was not lost in the L262P-mutant bearing cells grown for the time period of the experiment. However, a decrease in the levels of minimally edited MURF2 mRNA and pan-edited A6, ND8, and RPS12 RNAs was observed (Fig. 6D). The defect in editing of pan-edited RNAs was quite dramatic, with an almost two order of magnitude reduction in their levels when compared with the parental, L262P $\gamma$-subunit heterozygote controls. It should be noted that there was a concomitant accumulation of pre-edited mRNAs in the DKOs in relation to the parental cell lines that was on par with what was observed in PS when MRB8620 was down-regulated compared with the noninduced controls. To elaborate, pre-edited A6 in the BS DKO was 1.1-1.6 times the parental levels versus PS RNAi-induced (grown in SDM79) showing approximately two times the noninduced control levels for the same transcript. Similarly, RPS12 pre-edited in the BS DKO was 1.5-2.2 times the parental control compared to PS induced showing 1.2-1.3 times the noninduced level. Thus, it appears that RNA editing is compromised in $M R B 8620 \mathrm{DKO}$, indicating it plays a role in this process in BS.

\section{DISCUSSION}

We have studied in detail MRB8620, a subunit of the MRB1 core subcomplex that functions in trypanosome RNA editing. However, the findings we describe here are not just restricted to this subunit but also contribute to our understanding of the complex in general.

Disruption of MRB1 core integrity by MRB8620 knockdown leads to a reduction in mt RNA editing in T. brucei. The obvious phenotype in this condition is a down-regulation of edited mRNAs, which was observed in our study in both PS and BS, in contrast to previous work (Aphasizheva et al. 2014). Yet, impairment of editing also led to other consequences. For example, gRNAs exhibited an increase in their steady-state levels, as was observed when other MRB1 proteins were silenced by RNAi (Ammerman et al. 2013; Aphasizheva et al. 2014), suggesting that these small noncoding transcripts are consumed after fulfilling their guiding role during the normal, unimpeded course of RNA editing.

We did not observe any effect on doubling time in PS T. brucei when the MRB8620 knockdown was grown in SDM79 medium. Under these culture conditions, glycolysis seems to be sufficient to cover cellular ATP demands (Coustou et al. 2008). Thus, it is not surprising that the relatively modest effect we observed upon MRB8620 silencing on RNA editing, which is essential for the maturation of mRNAs encoding subunits of the respiratory chain, would not result in reduced fitness of flagellates that do not rely on oxidative phosphorylation for energy generation. This metabolic pathway rises to prominence under the low glucose condition of SDM80. Indeed, here we do observe a slight growth inhibition of PS upon MRB8620 silencing. This moderate effect on flagellate fitness may be due to the low pene- trance of RNAi in this study. Alternatively, the observed reduction in MRB1 complex integrity upon MRB8620 silencing may itself result in suboptimal editing. The other MRB1 proteins are still present in this background but are less efficient in their contribution to the process, which nevertheless occurs at sufficient levels to allow unaltered growth. MRB8620 may also be a particularly stable protein that persists at lower but still adequate doses during the course of RNAi silencing (i.e., residual MRB8620 still functions despite being diluted by RNAi targeting de novo protein during cell growth). We speculate that the finding that MRB8620 is membrane associated, and bears a predicted hydrophobic domain that may serve as a transmembrane domain, could also contribute to a possible higher degree of protein stability. The membrane association of MRB8620 would not be unprecedented, as other proteins involved in $T$. brucei $\mathrm{mt}$ RNA processing also exhibit this property. Several pentatricopeptide proteins, which are so named because they contain the eponymous RNA-binding motif, have been shown to be membrane associated along with $\mathrm{mt}$ ribosomal RNA (Pusnik et al. 2007). Among these is the kinetoplast polyadenylation/ uridylation factor 1 (kPAF1), also known as PPR1, which interacts with both MRB1 and $\mathrm{mt}$ ribosomes (Aphasizheva et al. 2011; Ammerman et al. 2012).

As demonstrated by the RNAi-mediated knockdown, MRB8620 plays a central role in MRB1 core integrity. This condition may be due to disrupted assembly of the constituent subunits during construction of the core and/or it may reflect a core that is especially unstable in the absence of MRB8620. Nevertheless, when MRB8620 is down-regulated, partner proteins GAP1, MRB11870, and MRB3010 exhibit a decreased associated with each other as a MRB1 core. The core's association with the TbRGG2 subcomplex, which appears to be the MRB1 component responsible for the processivity of pan-editing (Ammerman et al. 2010; Hashimi et al. 2013), is also reduced and its integrity somewhat disrupted upon MRB8620 depletion. The phenotype differs when the GAP1/2 heterotetramer is depleted by RNAi. In this case, MRB11870 interacts with MRB3010 to the same degree or more than in the untreated controls, implying that the MRB1 core remains intact despite the depletion of the GAP1/2 heterotetramer and the downstream effects manifested by gRNA degradation and pre-edited mRNA accumulation. Furthermore, association of the TbRGG2 subcomplex with the MRB1 core also persists, in agreement with its density gradient sedimentation remaining unperturbed upon GAP1 silencing (Aphasizheva et al. 2014), as well as its interaction with the core via MRB3010 or MRB8620, as demonstrated in a yeast two-hybrid screen (Ammerman et al. 2012). Taken together, these data indicate that MRB1 core integrity is dependent on subunits such as MRB8620 and MRB11870 (Ammerman et al. 2013), whereas the presence of the GAP $1 / 2$ heterotetramer is dispensable for its assembly or stability and even its association with TbRGG2. This finding plus the heterodispersion of the GAP1/2 subcomplex in 
density gradients (Acestor et al. 2009; Hashimi et al. 2009; Ammerman et al. 2011, 2012; Kafková et al. 2012; Aphasizheva et al. 2014) and the association of GAP1/2 with REH2 (Madina et al. 2014) or TbRGG3 (McAdams et al. 2015) suggest that GAP1/2 interactions are not restricted to the MRB1 core and that the heterotetramer represents a functionally distinct entity.

Another consequence of impaired MRB1 core fabrication is the accumulation of pre-edited $\mathrm{mRNAs}$ in association with the GAP1/2 heterotetramer. The two GAPs are the only established RBPs of the MRB1 core, as they have been shown to bind gRNAs to promote their stability, hence their aliases (Weng et al. 2008; Hashimi et al. 2009). This work also shows that the heterotetramer associates with pre-edited and edited mRNAs, although the nature of their interaction remains unknown (e.g., whether they are directly bound to the proteins or indirectly bound via gRNA hybridization or another bound protein partner). As the gRNA:mRNA duplex represents the substrate for RNA editing, it is plausible that GAP1/2 would pull down both transcript species. Upon disruption of the MRB1 core, pre-edited mRNAs that are slated for maturation via this process could become clogged on the GAP1/2 heterotetramer as they do not proceed along this pathway. Consistent with this hypothesis is the redistribution of GAP1 sedimentation throughout the glycerol gradient performed on samples from the MRB8620-silenced trypanosomes. The shift of GAP1 to more dense fractions upon MRB8620 silencing may reflect its increased accumulation on mRNA as described above. Because GAP1 associates with additional proteins outside the MRB1 complex (Madina et al. 2014; McAdams et al. 2015), these larger GAP1-containing complexes may also comprise non-MRB1 ribonucleoprotein complexes. Similar shifts of GAP1 sedimentation to larger glycerol gradient fractions has been reported previously in response to depletion of specific MRB1 proteins (Aphasizheva et al. 2014).

However, we observe that GAP1/2bound edited RNAs are present at the same level in both MRB8620-down-regulated and untreated controls, or even increased in the former when normalized to the reduced level of these transcripts when the whole mt transcriptome is concerned. One explanation is that the cDNAs annealing to what we term edited primers may not be fully edited. Another explanation that is not mutually exclusive to the previous one is that RNAi silencing does not fully deplete MRB8620, letting RNA editing occur at a lower level than normally. In this background, residual edited RNAs are enriched in association with the GAP heterotetramer. Supporting this finding is that KREL1, one of the RECC enzymes providing the machinery for $\mathrm{U}$ insertion/deletion, still associates with GAP1 when MRB8620 is down-regulated, although to a lesser degree as compared to the untreated control. In the untreated controls, the pool of edited mRNA sequence that is sequestered by GAP1/2 may represent only a small fraction of these transcripts. The residual amounts of edited RNA remaining in the MRB8620-depleted background could associate with GAP1/2 to a similar degree as in the controls, where edited RNAs may not reside long with MRB1 after completion of the editing process.

MRB1 has been interpreted to be a platform for the various components of the RNA editing and other RNA processes to assemble (Hashimi et al. 2013) or a vital part of the RNA editing holoenzyme along with RECC (Aphasizheva et al. 2014). Regardless of one's point of view, it is clear that cooperation among several protein subcomplexes is needed for RNA editing to work. In this study, we show that MRB8620 is required for proper assembly of the MRB1 core. A model arising from the investigated consequences of MRB1 core disassembly is shown in Figure 7, to suggest

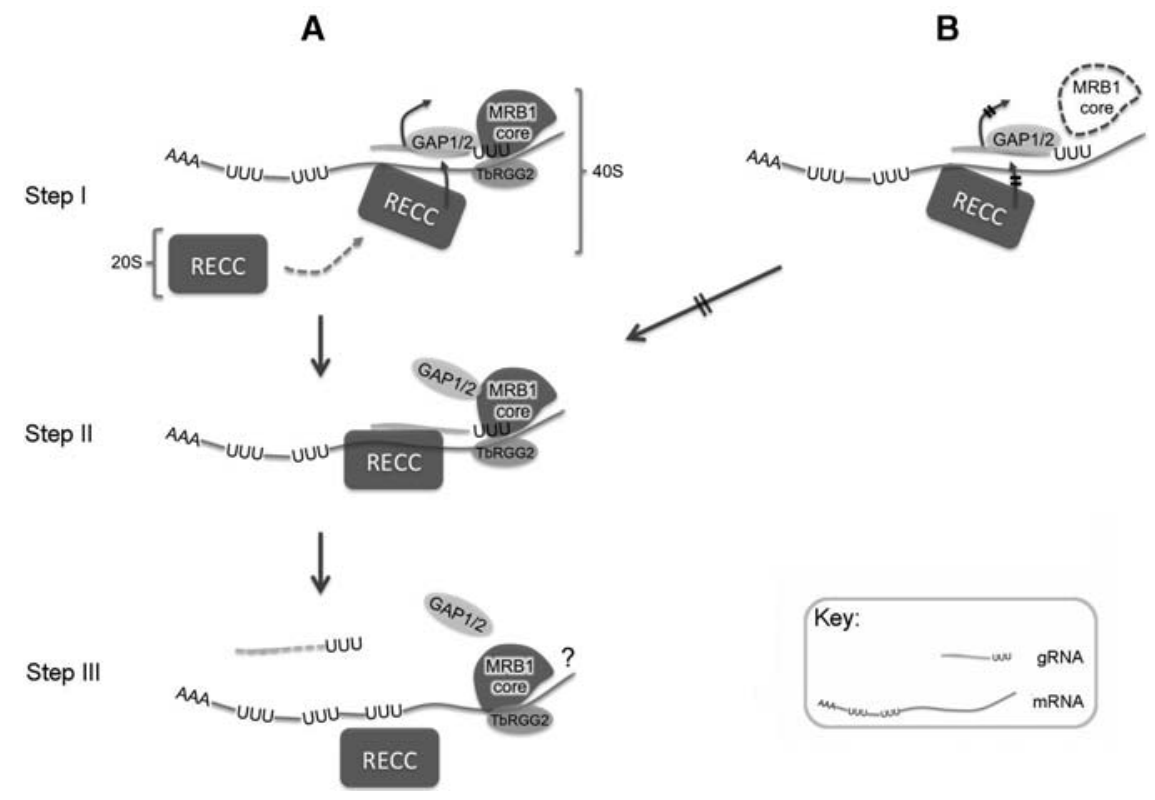

FIGURE 7. Proposed role of MRB1 core assembly in RNA editing. (A) Assembly of editing machinery on duplexed gRNA:mRNA in the native state of untreated T. brucei. $3^{\prime}$-Oligo(U) bearing gRNA strand on top with $3^{\prime}$-poly $(\mathrm{A} / \mathrm{U})$ tail appended, partially edited mRNA on bottom. Step I: 20 S RECC assembled with gRNA-bound GAP1/2 and MRB1 core as well as TbRGG2 subcomplex bound to mRNA to form $40 \mathrm{~S}$ particle. Arrows indicate conformational change removing GAP1/2 from gRNA to allow RECC access to editing site as defined by gRNA:mRNA duplex. Step II: RECC processes mRNA:gRNA free of obstructive GAP1/2 heterotetramer. Step III: gRNA is degraded after use in editing of prior mRNA/gRNA duplex. RECC and GAP1/2 assumed to disassociate. Question mark (?) indicates that MRB1 core and TbRGG2 remaining attached to still partially edited mRNA remains unknown. (B) Consequence of MRB1 core disassembly (dashed outline) caused by MRB8620 silencing on editing. Crossed arrows indicate that the conformational change freeing GAP1/2 from the gRNA:mRNA duplex, allowing RECC to bind to the hybridized transcripts, does not occur because of disassembly of MRB1 core. 
how this core subcomplex may serve to facilitate RNA editing.

We demonstrated that while GAP1/2 undoubtedly interacts with the MRB1 core, it may represent a separate entity that is functionally distinguishable from the other core subunits (Fig. 7A, Step I). Furthermore, when GAP1 is RNAi silenced, interaction between the core protein MRB3010 and TbRGG2 persists. Because gRNA and edited RNAs are depleted in this background, we presume the interaction between MRB3010 and TbRGG2 is dependent on proteinprotein interactions enhanced by the presence of pre-edited and/or partially edited mRNAs (Fig. 7A, Step I), consistent with previous results (Ammerman et al. 2012; Foda et al. 2012). Thus, our results suggest that the association of the MRB1 core with mRNA is facilitated by interaction with the TbRGG2 subcomplex (Fig. 7, Step I).

We have also shown that GAP1/2 associates with mRNA undergoing RNA editing, and that these transcripts appear to accumulate on the heterotetramer when MRB1 core assembly is disrupted. This observation is a further testament to GAP1/2 being functionally distinct to the MRB1 core. Decreased RECC association with GAP1/2 in this background is also consistent with the mRNA accumulation phenotype, because this protein complex provides the catalytic activities for the processing of these transcripts (Fig. 7B).

To explain these findings, we propose that in the native state, the MRB1 core may serve to facilitate a conformational change so that GAP1/2 is removed from the gRNA:mRNA duplex so that it does not sterically hinder RECC access to ESs along the hybridized transcripts (Fig. 7A, Steps I and II). It has been previously shown that although RECC sedimenting at $20 \mathrm{~S}$ fractions is depleted of RNAs, thus having its catalytic sites free for in vitro RNA editing assays, the heavier 40S RECC fractions do contain RNA and associate with GAP1/2 (Golas et al. 2009; Aphasizheva et al. 2014). Thus, we propose that although the GAP1/2 heterotetramer may be shifted away from a processed ES, it remains anchored to the mRNA undergoing processing via the MRB1 and TbRGG2 subcomplexes (Fig. 7A, Step II). In the MRB8620-depleted background, in which the MRB1 core cannot be properly assembled, mRNAs that undergo editing accumulate on GAP1/2 because RECC access to the gRNA: mRNA duplex is hindered (Fig. 7B).

GAP1/2 release from the RECC processed gRNA:mRNA duplex is also supported by the finding that gRNAs are degraded after they have been used by RNA editing (Fig. 7A, Step III; Ammerman et al. 2013; Aphasizheva et al. 2014), because the binding of these small RNAs to the heterotetramer promotes their stability (Weng et al. 2008; Hashimi et al. 2009). How the discussed protein complexes behave after editing of a block as defined by a gRNA:mRNA duplex remains unknown. RECC and the GAPs most likely dissociate from the processed RNA (Fig. 7A, Step III). Perhaps the MRB1 core remains associated with a partially edited mRNA to facilitate steps I and II as depicted in Figure 7A for the next round of editing. Collectively, the data presented here demonstrate an important role for the MRB1 core in RNA trafficking during kinetoplastid RNA editing.

\section{MATERIALS AND METHODS}

\section{Generation of cell lines and their manipulation}

A 575-bp-long portion of the $3^{\prime}$ part of the MRB8620 open reading frame excluding the stop codon was cloned into the pC-PURO-PTP vector, a variation on $\mathrm{pC}-\mathrm{NEO}-\mathrm{PTP}$, via the ApaI and NotI sites in the polylinker (Schimanski et al. 2005). This construct was linearized by cutting with SphI, whose single restriction site is within the MRB8620 sequence, to create homology flanks for in situ tagging of the gene product for affinity purification. Cells with proper integration of the construct were selected by their resistance to puromycin. In situ C-terminal tagging of MRB8620 and MRB3010 was facilitated by previously described vectors (Huang et al. 2014). To generate RNAi knockdowns, PCR primers were designed using the RNAit tool (Redmond et al. 2003) for amplifying an appropriate region of the MRB8620 ORF from T. brucei genomic DNA, for cloning into the p2T7-177 vector (Wickstead et al. 2002) allowing inducible RNAi. The linearized constructs were electroporated into the PS 29-13 strain by an established protocol (Kafková et al. 2012). Cell density in SDM79 (Brun and Schönenberger 1979) and in glucose-poor SDM80 (Coustou et al. 2008) was measured daily with a Coulter counter as described previously (Kafková et al. 2012).

For the double knockout of MRB8620 in the BS 427 strain, a kDNA-independent cell line was first generated by replacing an endogenous ATP synthase $F_{1} \gamma$-subunit locus with an ORF bearing the L262P mutation, which was selected with puromycin (Dean et al. 2013). To verify that the generated cell line was truly kDNA-independent, it was grown in the presence of $20 \mathrm{nM}$ ethidium bromide for $5 \mathrm{~d}$. The lack of 4',6-diamidino-2-phenylindole (DAPI) stained kDNA confirmed that the L262P $\gamma$-subunit mutation allowed survival despite loss of the organellar genome (data not shown). The MRB8620 knockout constructs were generated using a fusion PCR method (Merritt and Stuart 2013) to generate either the hygromycin or phleomycin resistance marker cassettes flanked by MRB8620 $5^{\prime}$ - and $3^{\prime}$ - untranslated regions to facilitate homologous recombination. These constructs were sequentially electroporated into the kDNA-independent BS T. brucei by an established protocol (Ammerman et al. 2013). The PCR primers amplifying elements incorporated into the described constructs used to generate the various cell lines are summarized in Supplemental Table 2.

\section{RNA detection assays}

RNA isolation and gRNA detection by probing of Northern blots with $5^{\prime}-\mathrm{P}^{32}$ labeled oligonucleotide probes (sequences are given in Supplemental Table 2) were conducted as described elsewhere (Kafková et al. 2012). Quantitative real-time PCR was also performed according to this study except the generation of template cDNA from the RNAi-silenced and knockout cells. The QuantiTech Reverse Transcription kit (QIAGEN) was used for generating cDNA from $4 \mu \mathrm{g}$ of RNA, with a prior genomic DNA degradation step, according to the manufacturer's instructions. Primers 
for detection of MRB8620 cDNA by qPCR are given in Supplemental Table 2. Other primers used in this study, along with a description of relative abundances of cDNAs between treated and untreated samples, were described previously (Carnes et al. 2005).

\section{Protein detection assays and cell fractionation}

Tandem affinity purification (TAP) of MRB8620 via its C-terminal PTP (ProtC-TEV-ProtA) (Schimanski et al. 2005) tag was performed following an established protocol (Ammerman et al. 2011) to yield eluates that were analyzed by LC-MS/MS mass spectroscopy as previously described (Kafková et al. 2012). The separation of mitochondria and cytosolic components, as well as further fractionation of the former into soluble and insoluble parts, was performed as described elsewhere (Schnaufer et al. 2005). In brief, $1 \times$ $10^{8}$ cells were treated with $1 \mathrm{~mL}$ of $0.015 \%$ digitonin in SoTE buffer (20 mM Tris, pH 7.5; 0.6 M sorbitol; and 2 mM EDTA) on ice and then separated by centrifugation into the cytosolic (supernatant) and organellar (pellet) fractions. The volume of the cytosolic fraction was measured and the pellet was resuspended in an equal volume of $1 \%$ Triton X-100 and separated into soluble (supernatant) and insoluble (pellet) fractions by centrifugation; the pellet was again resuspended in the same volume as the supernatant. Equal volumes from each fraction, representing the same proportion of each fraction, was loaded onto a SDS-PAGE gel along with a cell equivalent of total lysate from T. brucei.

\section{Glycerol gradients and immunoprecipitation}

Mitochondria were isolated from $10^{10}$ MRB8620 and GAP RNAi knockdown and parental control flagellates as described previously (Hashimi et al. 2008). The resulting vesicles corresponding to $5 \mathrm{mg}$ of protein were lysed in $1 \mathrm{~mL}$ of the lysis buffer $(10 \mathrm{mM}$ Tris at $\mathrm{pH}$ 7.2, $10 \mathrm{mM} \mathrm{MgCl}_{2}, 100 \mathrm{mM} \mathrm{KCl}, 1 \mathrm{mM} \mathrm{DTT}, 1 \mathrm{mg} / \mathrm{mL}$ pepstatin, and $2 \mathrm{mg} / \mathrm{mL}$ leupeptin) with $1 \%$ Triton X-100 and treated with $20 \mathrm{U}$ RNaseOUT ${ }^{\mathrm{TM}}$ (Life Technology) for $60 \mathrm{~min}$ at $4^{\circ} \mathrm{C}$. The lysate was cleared and loaded on an 11-mL 10\%-30\% glycerol gradient, which was centrifuged at $32,000 \mathrm{rpm}$ in a Beckman SW41 rotor for $16 \mathrm{~h}$ at $4^{\circ} \mathrm{C}$. Twelve $0.5-\mathrm{mL}$ fractions were then collected from the top of the gradient for Western blot analysis.

Mitochondria from $2 \times 10^{9}$ MRB3010-V5 PS cells were harvested and lysed as for the glycerol gradients. IP of MRB3010-YFP was performed as previously described (Huang et al. 2014), and elutions were analyzed by Western blotting. Equal volumes from the IP eluates generated from an equal amount of cells were loaded onto $12 \%$ or $15 \%$ SDS-polyacrylamide gels for the resolution of the constituent proteins.

The cross-linking of $\alpha$-GAP1 antibody to Protein G Dynabeads (Life Technology) was done with dimethyl pimelimidate (DMP) (Sigma-Aldrich) as follows. Beads were washed two times with phosphate-buffered saline (PBS) at 1:1 ratio by rotation for $10 \mathrm{~min}$ at $4^{\circ} \mathrm{C}$. After discarding the supernatant, dilution buffer $(\mathrm{PBS}+1 \mathrm{mg} / \mathrm{mL}$ BSA $)$ was added at 1:1 ratio and rotated for 10 min at $4^{\circ} \mathrm{C}$. Approximately $20 \mu \mathrm{g}$ of $\alpha$-GAP1 antibody in dilution buffer was then added at a 1:1 ratio to the beads and rotated $1 \mathrm{~h}$ at $4^{\circ} \mathrm{C}$. Afterward, the beads were washed again in PBS as before. Thirteen milligrams per milliliter DMP solution $\mathrm{pH} 8$ was prepared immediately prior to cross-linking and mixed with equal volume of
0.2 M triethanolamine in PBS. The cross-linking solution was added to the beads at a 1:1 ratio and rotated for $30 \mathrm{~min}$ at room temperature (RT). Then the beads were washed three times with PBS as before. A quenching buffer of $50 \mathrm{mM}$ ethanolamine in PBS was added to beads at a 1:1 ratio and rotated $5 \mathrm{~min}$ at RT three times and then washed with PBS as before. The beads were then treated twice with 1 $\mathrm{M}$ glycine $\mathrm{pH} 3$ and then rotated for $10 \mathrm{~min}$ at RT. The beads were then ready for IP after a last PBS wash. RNA IP was performed as the MRB3010-YFP IP, with the addition of a preclearing step by gentle agitation with Protein G Dynabeads for $30 \mathrm{~min}$ and three subsequent stringent washing steps (10 mM Tris, $\mathrm{pH}$ 7.4; 1 mM EDTA; $1 \mathrm{mM}$ EGTA; $\mathrm{NaCl} 300 \mathrm{mM}$; and 1\% Triton X-100) for reduction of background. RNA from the IP was recovered by acid phenolchloroform extraction and a successive ethanol precipitation. The RNA IP was performed two times. RNAs were then processed for the qPCR assay for mt transcript levels as described above and performed in technical triplicates.

The formula for normalization of RNA IP to mt RNA levels in the MRB8620 knockdown (Fig. 3A) is as follows: $V_{\text {IP }}$, representing abundance value of one tested transcript from the elution, $V_{\mathrm{T}}$ as the same transcript from the lysis, relative GAP1/2-associated mRNA fraction value $V_{\mathrm{f}}=V_{\mathrm{IP}} / V_{\mathrm{T}}$, the GAP1/2-associated mRNA fraction fold number $R_{\mathrm{f}}=\left(V_{\mathrm{IP} \text {-induced }} / V_{\mathrm{T} \text {-induced }}\right) /\left(V_{\mathrm{IP}-\text { noninduced }} /\right.$ $\left.V_{\text {T-noninduced }}\right)=\left(V_{\text {IP-induced }} / V_{\text {IP-noninduced }}\right) /\left(V_{\text {T-induced }} / V_{\text {T-noninduced }}\right)$ $=(2 \times \mathrm{PCR} \text { efficiency percent })^{(\text {CtIPnoninduced-CtIPinduced })} /(2 \times \mathrm{PCR}$ efficiency percent $)^{(\text {Ctnon-induced-Ctinduced })}$.

\section{Western blot analysis and antibodies}

Forty micrograms of proteins from hypotonically isolated mitochondrial vesicles were loaded on $12 \%$ or $15 \%$ SDS-polyacrylamide gels and then transferred to a PVDF membrane. Western blot analysis was performed with the following polyclonal antibodies, all at 1:1000: $\alpha$-MRB3010, $\alpha$-MRB11870, and $\alpha$-MRB8170 (Ammerman et al. 2012); $\alpha$-GAP1 (Hashimi et al. 2009); $\alpha$-TbRGG2 (Fisk et al. 2008); $\alpha$-trCoIV (Maslov et al. 2002); a-protein A (SigmaAldrich); and $\alpha-\mathrm{V} 5$ (Life Technologies). Anti-enolase antibody was used at a 1:10,000 dilution. In addition, monoclonal antibodies immunodecorating KREL1 and KREPA3 (Panigrahi et al. 2001) were also used at 1:50 dilutions.

\section{SUPPLEMENTAL MATERIAL}

Supplemental material is available for this article.

\section{ACKNOWLEDGMENTS}

We thank Achim Schnaufer (University of Edinburgh) for providing constructs and protocols for creating the kDNA-independent BS cell line used in this study, Ken Stuart (Seattle Biomed) and Paul Michels (Universidad de los Andes/University of Edinburgh) for the kind gift of antibodies, and Peter Koník (University of South Bohemia) for help with mass spectroscopy. This work was supported by the Grant Agency of the Czech Republic (15-21974S), RNPnet FP7 program (289007), and Praemium Academiae to J.L., and the National Institutes of Health (NIH) (RO1 AI061580) to L.K.R.

Received April 24, 2015; accepted September 9, 2015. 


\section{REFERENCES}

Acestor N, Panigrahi AK, Carnes J, Zíková A, Stuart KD. 2009. The MRB1 complex functions in kinetoplastid RNA processing. RNA 15: $277-286$.

Ammerman ML, Presnyak V, Fisk JC, Foda BM, Read LK. 2010. TbRGG2 facilitates kinetoplastid RNA editing initiation and progression past intrinsic pause sites. RNA 16: 2239-2251.

Ammerman ML, Hashimi H, Novotná L, Cičová Z, McEvoy SM, Lukeš J, Read LK. 2011. MRB3010 is a core component of the MRB1 complex that facilitates an early step of the kinetoplastid RNA editing process. RNA 17: 865-877.

Ammerman ML, Downey KM, Hashimi H, Fisk JC, Tomasello DL, Faktorová D, Kafková L, King T, Lukeš J, Read LK. 2012. Architecture of the trypanosome RNA editing accessory complex, MRB1. Nucleic Acids Res 40: 5637-5650.

Ammerman ML, Tomasello DL, Faktorová D, Kafková L, Hashimi H, Lukeš J, Read LK. 2013. A core MRB1 complex component is indispensable for RNA editing in insect and human infective stages of Trypanosoma brucei. PLoS One 8: e78015.

Aphasizheva I, Maslov D, Wang X, Huang L, Aphasizhev R. 2011. Pentatricopeptide repeat proteins stimulate mRNA adenylation/uridylation to activate mitochondrial translation in trypanosomes. $\mathrm{Mol}$ Cell 42: 106-117.

Aphasizheva I, Zhang L, Wang X, Kaake RM, Huang L, Monti S, Aphasizhev R. 2014. RNA binding and core complexes constitute the U-insertion/deletion editosome. Mol Cell Biol 34: 4329-4342.

Blum B, Bakalara N, Simpson L. 1990. A model for RNA editing in kinetoplastid mitochondria: "guide" RNA molecules transcribed from maxicircle DNA provide the edited information. Cell 60: 189-198.

Brown SV, Hosking P, Li J, Williams N. 2006. ATP synthase is responsible for maintaining mitochondrial membrane potential in bloodstream form Trypanosoma brucei. Eukaryot Cell 5: 45-53.

Brun R, Schönenberger. 1979. Cultivation and in vitro cloning or procyclic culture forms of Trypanosoma brucei in a semi-defined medium. Acta Trop 36: 289-292.

Carnes J, Trotter JR, Ernst NL, Steinberg A, Stuart K. 2005. An essential RNase III insertion editing endonuclease in Trypanosoma brucei. Proc Natl Acad Sci 102: 16614-16619.

Carnes J, Trotter JR, Peltan A, Fleck M, Stuart K. 2008. RNA editing in Trypanosoma brucei requires three different editosomes. Mol Cell Biol 28: 122-130.

Coustou V, Biran M, Breton M, Guegan F, Rivièere L, Plazolles N, Nolan D, Barrett MP, Franconi JM, Bringaud F. 2008. Glucose-induced remodeling of intermediary and energy metabolism in procyclic Trypanosoma brucei. J Biol Chem 283: 16342-16354.

Dean S, Gould MK, Dewar CE, Schnaufer AC. 2013. Single point mutations in ATP synthase compensate for mitochondrial genome loss in trypanosomes. Proc Natl Acad Sci 110: 14741-14746.

Ernst NL, Panicucci B, Igo RP Jr, Panigrahi AK, Salavati R, Stuart K. 2003. TbMP57 is a $3^{\prime}$ terminal uridylyl transferase (TUTase) of the Trypanosoma brucei editosome. Mol Cell 11: 1525-1536.

Ernst NL, Panicucci B, Carnes J, Stuart K. 2009. Differential functions of two editosome exoUases in Trypanosoma brucei. RNA 15: 947-957.

Fisk JC, Ammerman ML, Presnyak V, Read LK. 2008. TbRGG2, an essential RNA editing accessory factor in two Trypanosoma brucei life cycle stages. J Biol Chem 283: 23016-23025.

Foda BM, Downey KM, Fisk JC, Read LK. 2012. Multifunctional G-rich and RRM-containing domains of TbRGG2 perform separate yet essential functions in trypanosome RNA editing. Eukaryot Cell 11: 1119-1131.

Golas MM, Böohm C, Sander B, Effenberger K, Brecht M, Stark H, Göoringer HU. 2009. Snapshots of the RNA editing machine in trypanosomes captured at different assembly stages in vivo. EMBO J 28: 766-778.

Golden DE, Hajduk SL. 2005. The 3'-untranslated region of cytochrome oxidase II mRNA functions in RNA editing of African trypanosomes exclusively as a cis guide RNA. RNA 11: 29-37.
Hashimi H, Zíková A, Panigrahi AK, Stuart KD, Lukeš J. 2008. TbRGG1, an essential protein involved in kinetoplastid RNA metabolism that is associated with a novel multiprotein complex. RNA 14: 970-980.

Hashimi H, Čičová Z, Novotná L, Wen YZ, Lukeš J. 2009. Kinetoplastid guide RNA biogenesis is dependent on subunits of the mitochondrial RNA binding complex 1 and mitochondrial RNA polymerase. RNA 15: 588-599.

Hashimi $\mathrm{H}$, Benkovičová $\mathrm{V}$, Čermáková $\mathrm{P}$, Lai $\mathrm{DH}$, Horváth $\mathrm{A}$, Lukeš J. 2010. The assembly of $\mathrm{F}_{1} \mathrm{~F}_{\mathrm{O}}$-ATP synthase is disrupted upon interference of RNA editing in Trypanosoma brucei. Int J Parasitol 40: $45-54$.

Hashimi H, Zimmer SL, Ammerman ML, Read LK, Lukeš J. 2013. Dual core processing: MRB1 is an emerging kinetoplast RNA editing complex. Trends Parasitol 29: 91-99.

Hernandez A, Madina BR, Ro K, Wohlschlegel JA, Willard B, Kinter MT, Cruz-Reyes J. 2010. REH2 RNA helicase in kinetoplastid mitochondria: ribonucleoprotein complexes and essential motifs for unwinding and guide RNA (gRNA) binding. J Biol Chem 285: $1220-1228$.

Huang Z, Kaltenbrunner S, Šimková E, Staněk D, Lukeš J, Hashimi H. 2014. Dynamics of mitochondrial RNA-binding protein complex in Trypanosoma brucei and its petite mutant under optimized immobilization conditions. Eukaryot Cell 13: 1232-1240.

Kafková L, Ammerman ML, Faktorová D, Fisk JC, Zimmer SL, Sobotka R, Read LK, Lukeš J, Hashimi H. 2012. Functional characterization of two paralogs that are novel RNA binding proteins influencing mitochondrial transcripts of Trypanosoma brucei. RNA 18: $1846-1861$.

Lai DH, Hashimi H, Lun ZR, Ayala FJ, Lukeš J. 2008. Adaptations of Trypanosoma brucei to gradual loss of kinetoplast DNA Trypanosoma equiperdum and Trypanosoma evansi are petite mutants of T. brucei. Proc Natl Acad Sci 105: 1999-2004.

Madina BR, Kumar V, Metz R, Mooers BHM, Bundschuh R, CruzReyes J. 2014. Native mitochondrial RNA-binding complexes in kinetoplastid RNA editing differ in guide RNA composition. RNA 20: $1142-1152$.

Maslov DA, Simpson L. 1992. The polarity of editing within a multiple gRNA-mediated domain is due to formation of anchors for upstream gRNAs by downstream editing. Cell 70: 459-467.

Maslov DA, Zíková A, Kyselová I, Lukeš J. 2002. A putative novel nuclear-encoded subunit of the cytochrome $c$ oxidase complex in trypanosomatids. Mol Biochem Parasitol 125: 113-125.

McAdams NM, Ammerman ML, Nanduri J, Lott K, Fisk JC, Read LK. 2015. An arginine-glycine-rich RNA binding protein impacts the abundance of specific mRNAs in the mitochondria of Trypanosoma brucei. Eukaryot Cell 14: 149-157.

McManus MT, Adler BK, Pollard VW, Hajduk SL. 2000. Trypanosoma brucei guide RNA poly $(\mathrm{U})$ tail formation is stabilized by cognate mRNA. Mol Cell Biol 20: 883-891.

Merritt C, Stuart K. 2013. Identification of essential and non-essential protein kinases by a fusion PCR method for efficient production of transgenic Trypanosoma brucei. Mol Biochem Parasitol 190: $44-49$.

Möller S, Croning MD, Apweiler R. 2001. Evaluation of methods for the prediction of membrane spanning regions. Bioinformatics 17: 646653.

Neboháčová M, Maslov DA, Falick AM, Simpson L. 2004. The effect of RNA interference down-regulation of RNA editing $3^{\prime}$-terminal uridylyl transferase (TUTase) 1 on mitochondrial de novo protein synthesis and stability of respiratory complexes in Trypanosoma brucei.J Biol Chem 279: 7819-7825.

Nilsson D, Gunasekera K, Mani J, Osteras M, Farinelli L, Baerlocher L, Roditi I, Ochsenreiter T. 2010. Spliced leader trapping reveals widespread alternative splicing patterns in the highly dynamic transcriptome of Trypanosoma brucei. PLoS Pathog 6: e1001037.

Panigrahi AK, Schnaufer A, Carmean N, Igo RP, Jr, Gygi SP, Ernst NL, Palazzo SS, Weston DS, Aebersold R, Salavati R, et al. 2001. Four 
related proteins of the Trypanosoma brucei RNA editing complex. Mol Cell Biol 21: 6833-6840.

Pfaffl MW. 2001. A new mathematical model for relative quantification in real-time RT-PCR. Nucleic Acids Res 29: e45.

Pusnik M, Small I, Read LK, Fabbro T, Schneider A. 2007. Pentatricopeptide repeat proteins in Trypanosoma brucei function in mitochondrial ribosomes. Mol Cell Biol 27: 6876-6888.

Redmond S, Vadivelu J, Field MC. 2003. RNAit: an automated webbased tool for the selection of RNAi targets in Trypanosoma brucei. Mol Biochem Parasitol 128: 115-118.

Schimanski B, Nguyen TN, Guünzl A. 2005. Highly efficient tandem affinity purification of trypanosome protein complexes based on a novel epitope combination. Eukaryot Cell 4: 1942-1950.

Schnarwiler F, Niemann M, Doiron N, Harsman A, Kaäser S, Mani J, Chanfon A, Dewar CE, Oeljeklaus S, Jackson CB, et al. 2014. Trypanosomal TAC40 constitutes a novel subclass of mitochondrial $\beta$-barrel proteins specialized in mitochondrial genome inheritance. Proc Natl Acad Sci 111: 7624-7629.

Schnaufer A, Panigrahi AK, Panicucci B, Igo RP Jr, Wirtz E, Salavati R, Stuart K. 2001. An RNA ligase essential for RNA editing and survival of the bloodstream form of Trypanosoma brucei. Science 291: 2159-2162.
Schnaufer A, Clark-Walker GD, Steinberg AG, Stuart K. 2005. The F1ATP synthase complex in bloodstream stage trypanosomes has an unusual and essential function. EMBO J 24: 4029-4040.

Siegel TN, Hekstra DR, Wang X, Dewell S, Cross GA. 2010. Genomewide analysis of mRNA abundance in two life-cycle stages of Trypanosoma brucei and identification of splicing and polyadenylation sites. Nucleic Acids Res 38: 4946-4957.

Verner Z, Basu S, Benz C, Dixit S, Dobáková E, Faktorová D, Hashimi H, Horáková E, Huang Z, Paris Z, et al. 2015. Malleable mitochondrion of Trypanosoma brucei. Int Rev Cell Mol Biol 315: 73-151.

Weng J, Aphasizheva I, Etheridge RD, Huang L, Wang X, Falick AM, Aphasizhev R. 2008. Guide RNA-binding complex from mitochondria of trypanosomatids. Mol Cell 32: 198-209.

Wickstead B, Ersfeld K, Gull K. 2002. Targeting of a tetracycline-inducible expression system to the transcriptionally silent minichromosomes of Trypanosoma brucei. Mol Biochem Parasitol 125: 211-216.

Zíková A, Horáková E, Jirků M, Dunajčíková P, Lukeš J. 2006. The effect of down-regulation of mitochondrial RNA-binding proteins MRP1 and MRP2 on respiratory complexes in procyclic Trypanosoma brucei. Mol Biochem Parasitol 149: 65-73. 

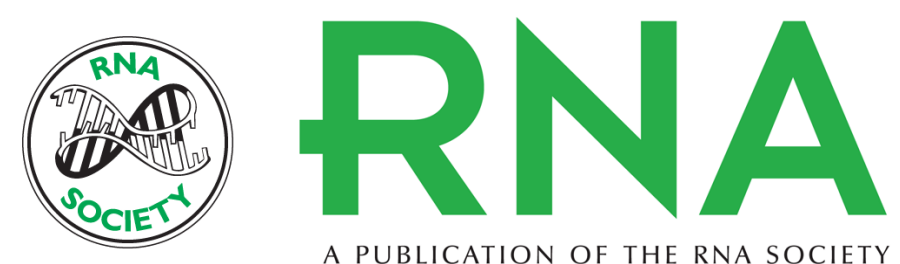

A PUBLICATION OF THE RNA SOCIETY

\section{Integrity of the core mitochondrial RNA-binding complex 1 is vital for trypanosome RNA editing}

Zhenqiu Huang, Drahomíra Faktorová, Adéla Krízová, et al.

RNA 2015 21: 2088-2102 originally published online October 7, 2015

Access the most recent version at doi:10.1261/rna.052340.115

\section{Supplemental http://rnajournal.cshlp.org/content/suppl/2015/10/02/rna.052340.115.DC1 Material}

References This article cites 50 articles, 30 of which can be accessed free at: http://rnajournal.cshlp.org/content/21/12/2088.full.html\#ref-list-1

Creative This article is distributed exclusively by the RNA Society for the first 12 months after the Commons License full-issue publication date (see http://rnajournal.cshlp.org/site/misc/terms.xhtml). After 12 months, it is available under a Creative Commons License (Attribution-NonCommercial 4.0 International), as described at http://creativecommons.org/licenses/by-nc/4.0/.

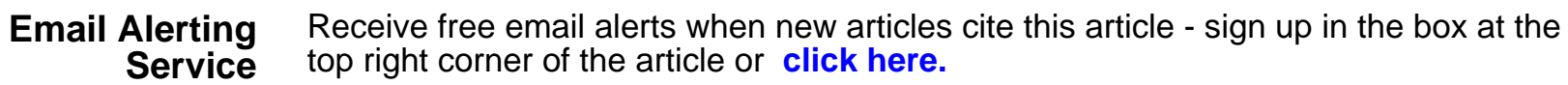

